\title{
Concentración de la propiedad de los medios de comunicación en Chile. La compleja relación entre oligopolio y democracia
}

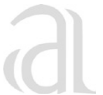 \\ Mg. Alberto Javier Mayorga Rogel \\ Dr. Carlos del Valle Rojas \\ Mg. Luis Nitrihual Valdebenito
}

Recibido: 10 de septiembre de 2010 Aceptado: 5 de octubre de 2010

\begin{abstract}
Resumen
En el presente trabajo se desarrolla una descripción de la situación actual en materia de concentración mediática en Chile, a partir de los datos obtenidos por el Instituto Prensa y Sociedad (IPyS) en el marco de una investigación desarrollada entre el 2002 y 2005. Así, entonces, se establece que la lógica de la concentración de los medios de comunicación social en Chile apunta a mejorar los procesos de control de información, fortalecer la posición en el mercado, incrementar el poder económico y político, estandarizar los contenidos y aumentar los ingresos mediante el consumo masivo de los productos culturales que circulan en el mercado interno.
\end{abstract}

Palabras clave: economía política, medios de comunicación, concentración económica, mercado infocomunicacional.

* $\quad$ Este artículo de investigación científica y tecnológica hace parte de la investigación "Concentración de la propiedad de los medios de comunicación en Chile. La compleja relación entre oligopolio y democracia", patrocinada por la Universidad de la Frontera, Temuco, Chile.

** Académico de la carrera de Periodismo y del magíster en Ciencias de la Comunicación. Departamento de Lenguas, Literatura y Comunicación. Facultad de Educación y Humanidades. Universidad de La Frontera. Temuco - Chile. Doctorando en Ciencia Política. Becario Fundación Volcán Calbuco. Dirección electrónical: Correo electrónico: amayorga@ufro.cl

*** Doctor en Periodismo, Comunicación e Información de la Universidad de Sevilla. Magíster en Ciencias de la Comunicación, Universidad de La Frontera. Licenciado en Comunicación Social, Universidad de La Frontera. Periodista, Universidad de la Frontera. Decano de la Facultad de Educación y Humanidades. Universidad de La Frontera. Temuco - Chile. Dirección electrónica: delvalle@ufro.cl

**** Académico de la carrera de Periodismo y del magíster en Ciencias de la Comunicación. Departamento de Lenguas, Literatura y Comunicación. Facultad de Educación y Humanidades. Universidad de La Frontera. Temuco - Chile. Becario del Fondo Nacional del Libro y La Lectura para estudios de postgrado en el extranjero 2008. Dirección electrónica: anitrihual@ufro.cl 


\title{
Communication media power concentration in Chile. A complex relationship between oligopoly and democracy
}

\begin{abstract}
The present work develops a description of the current situation of the mass media concentration in Chile, description based in the information obtained by the Presses and Society Institute (IPyS) in the frame of an investigation developed between 2002 and 2005. So it establish that the logic of the mass media concentration in Chile points out to improve the control information process, to strengthen the position on the market, to increase the economic and political power, to standardize the contents and to increase the income by the massive cultural products consumption that circulate on the internal market.
\end{abstract}

Key words: political economy, mass media, economic concentration, infomedia market. 


\section{Introducción}

En diversas sociedades el panorama de la concentración, dentro de la dinámica que se establece en el juego del sistema neoliberal, parece tomar fuerza en el área productiva de los medios de comunicación y en la conformación de oligopolios en mercados nacionales e internacionales.

Por tal motivo, podemos afirmar que las acciones comerciales de las empresas transnacionales de la información buscan accionar una penetración directa en los mercados periféricos, lo que produce una fragmentación de los colectivos en vías de desarrollo y, por lo tanto, fomentan la marginación de las empresas informativas locales como único mecanismo que evita la autogestión de los contenidos informativos, la reafirmación de identidades propias, la toma de conciencia acerca de la inoculación de ideologías dominantes y la movilización de grupos humanos capaces de fomentar un régimen democrático sustentable en el tiempo.

La incorporación de grupos económicos internacionales en el ámbito de las industrias culturales latinoamericanas -específicamente en el sector productivo de la comunicación y la información- apunta a la inversión de capitales que permita fortalecer la capacidad de gestión de recursos humanos, incorporación de nuevas tecnologías, actualización de los contenidos y control de la propiedad, en el caso de las empresas informativas.

Entonces, bajo esta lógica neoliberal que potencia la concentración de los medios de comunicación social, podemos aducir que la democracia, la política y la ciudadanía están siendo redefinidas desde las lógicas del consumo, la oferta y la demanda de productos mediatizados por los sistemas interconectados de información global. De esta manera, el argumento favorable a la situación mencionada apunta a la eficiencia que posee el mercado en el uso de los recursos, lo que sería -en la actualidad-incompatible con su deficiente habilidad en la distribución equitativa de los ingresos. Esto implica, según Norbert Lechner (2007), un debilitamiento de la comunidad de los ciudadanos y la redefinición del campo de lo político y el Estado, puesto que las dinámicas actuales de las relaciones de poder económico (mercado) por sobre la acción de los sistemas informativos (medios de comunicación), del hombre (ciudadanos) y las instituciones (Estado) conlleva una resemantización del espacio público y, en consecuencia, una transformación progresiva del hommo pûblicus en hommo videns.

La televisión escenifica la política acorde a sus reglas, modificando el carácter del espacio público. Por otra parte, produce una nueva mirada a la política (...) Usando un lenguaje audiovisual, el look del político puede crear mayor credibilidad que un buen argumento. Más la imagen es siempre ambigua, abierta a múltiples lecturas; requiere un anclaje que fije el mensaje. Aún así, la política llevada a la pantalla depende del contexto: el significado resulta fragmentado y recompuesto a través de la secuencia de imágenes como del contexto cotidiano del espectador (Lechner, 2007: 330).

Al señalar la necesidad de reflexionar en torno al problema de la concentración económica de los medios de comunicación social y su responsabilidad en el debilitamiento de la democracia en América Latina, se parte del supuesto que aduce una incapacidad de los medios de comunicación para colaborar en el fortalecimiento de una masa crítica a partir de la ausencia de contenidos centrados en temáticas de real interés público y, por supuesto, donde el núcleo de una sociedad democrática (espacio público, ciudadanía y sociedad civil) se ve afectado tanto por el declive de la información como bien público producto de la marginalidad adscrita al capital cultural como por la rápida concentración de esta industria estratégica en manos de las elites económicas.

En consecuencia, en el siguiente apartado nos enfocaremos en desarrollar conceptos clave para comprender las lógicas de concentración y conformación de oligopolios mediáticos desde la perspectiva de la economía política de la comunicación y, por último, se establecerá una descripción de la situación actual en materia de concentración mediática en Chile, a partir de los datos obtenidos por el Instituto Prensa y Sociedad (IPyS) en el marco de una investigación desarrollada entre el 2002 y 2005. 


\section{Oligopolios y democracia: una aproximación al fenómeno de la concentración de la propiedad mediática en América Latina}

En un régimen democrático contemporáneo, no se puede obviar la importancia social que tienen los medios de comunicación social y, mucho menos, negar la relación que existe entre la industria informativa y el sistema económico neoliberal imperante en América Latina. Esta relación puede ser vista desde la condición asimétrica del poder que mantienen los grupos económicos dominantes y que les permite ejercer una configuración política de la sociedad, es decir, mientras exista un control de las formas de producción informativa es plausible pensar en la configuración de un pacto social aparente en virtud del consenso impuesto a través de las redes internacionales de información.

Este último punto nos parece relevante en el debate actual sobre el rol de los medios de comunicación social en la democracia política latinoamericana. El poder que ostentan los conglomerados informativos (Red Televisa, Grupo Cisneros, Grupo Clarín, Grupo Edwards, Grupo Prisa, entre otros) en la actualidad se puede explicar mediante la identificación de sus respectivas formas de adaptación a las lógicas de los mercados internacionales y que versan en torno a una diversificación en distintas escalas de la cadena de valor y a la fusión de las empresas informativas, líderes en sus respectivos países, con aquellos grupos de comunicación e información mundial (Mastrini y Becerra, 2001).

De esta forma, los avances acaecidos en el proceso de la industrialización de la empresa informativa local/global permiten la entrada en el mercado mundial de un producto de consumo rápido y de naturaleza ideológica que conlleva la simulación de la integración del ciudadano-cliente al proceso de intercambio simbólico. No obstante, al negar la participación activa de los ciudadanos en la configuración de los contenidos, el mercado se encarga de potenciar una plataforma de intercambio entre los productores y los consumidores sobre la base de una reciprocidad correspondida entre las fuerzas de la oferta y la demanda (Torres López, 1985).

El fundamento de este argumento se sustenta en la dificultad que existe en América Latina y el mundo para promover la creación de medios de comunicación independientes a escala global/ nacional y, en consecuencia, a los ciudadanoscliente les resta participar en el mercado a través de la acción de compra y consumo acrítico de los bienes informativos que se producen y distribuyen por el sistema nacional e internacional de información.

En realidad, la participación de los ciudadanos-cliente que logran formar parte del proceso de intercambio en el marco de un mercado regulador de los productos informativos circulantes, se enfrenta a una transacción asimétrica producto de una entrada al sistema bajo un factor de desigualdad centrado en los recursos de poder del que dispone cada una de las partes involucradas en el juego de la producción de bienes y el consumo de éstos.

Para clarificar aún mejor esta afirmación, nos remitiremos a lo dicho por Giovanni Sartori:

Admitámoslo sin tapujos; el mercado es una entidad cruel. Su ley es la del éxito del mejor. Se dedica a encontrar un puesto adaptado a cada uno y se dedica a motivar en los individuos el máximo esfuerzo. Pero los irremediables inadaptados son expulsados de la sociedad del mercado y dejados perecer o sobrevivir de otros recursos. ¿A quién o a qué se imputa dicha crueldad? (...). La objeción de rigor es que el mercado y su ley de la competencia valen para los peces pequeños o medios, no para las multinacionales y los supercapitalistas: los grandes, y sobre todo los grandísimos, controlan o por lo general circundan el mercado y acaban con la competencia (2002: 158).

Podremos decir entonces que la ausencia de una competencia real, en un mercado como el de la información y la comunicación, no rompe la barrera de la fijación de precios por parte del capitalista-productor sino más bien elimina la capacidad de establecer un posible equilibrio de fuerzas ideológicas pero sin trastocar la operati- 
vidad de la estructura (mercado) vista como un mecanismo necesario para incentivar la productividad y, por supuesto, para la fijación de precios y costos (Sartori, 2002).

Por lo demás, el monopolio no es viable en el sector productivo de la información; por el contrario, como afirma Torres López (1985), el oligopolio es la situación más frecuente que se observa en el mercado de la comunicación, puesto que la ausencia de competencia atenta contra el crecimiento proyectado por la industria cultural (empresas informativas) y, en alguna medida (a modo de supuesto), puede poner en riesgo el control de la propiedad y la renovación continua de sus productos.

$\mathrm{Si}$ atendemos a las características centrales del fenómeno analizado, podemos asegurar, sobre la base de lo planteado por Ramón Zallo (1992: 107-108), que existen tres formas clásicas de concentración de capitales:

1. La integración vertical. Esta acción se ejecuta en las distintas fases de la producción, comenzando en las materias primas y finalizando en uno o varios productos acabados. Con este modelo de integración se pretende disminuir los costos, mejorar el uso de sinergias, aumentar la seguridad en el aprovisionamiento y el reforzamiento de la posición en el mercado.

2. La integración horizontal. Consiste en la producción de una variedad de productos finales de una misma rama. Aquí se potencia la elaboración y distribución de un conjunto de productos mediante las sinergias comerciales y el prestigio de la marca.

3. La integración en conglomerados. Se entiende como la capacidad de diversificar la inversión y la producción en varios sectores como una forma de reducir y compensar riesgos y aprovechar la oportunidad de producir durante un tiempo específico un producto determinado. Los conglomerados tienen una tendencia financiera que apunta a la entrada en sectores productivos por medio de la compra directa, fusión o absorción de empresas informativas.
Está claro, por lo tanto, que el panorama de la concentración de la propiedad de los medios de comunicación social es el reflejo de un desequilibrio en la distribución de la propiedad mediática que viene dado, según Flammant (citado en Torres López, 1985: 101), por 4 razones de carácter económico:

1. El carácter de las técnicas necesarias para la producción, muy costosa, así como el del personal altamente cualificado que ha de utilizarse.

2. La exigencia de considerables capitales financieros para el inicio y pervivencia de la actividad informativa.

3. La regularidad con que viene produciéndose la concentración financiera en el sector.

4. La influencia de la publicidad, al convertirse los ingresos por este concepto en base de supervivencia de los medios de comunicación de masas.

Estas circunstancias han provocado, por una parte, la comercialización a gran escala de una diversidad de productos informativos (noticias, publicidad, entretenimiento, propaganda política, entre otros) que el consumidor puede seleccionar dentro de los límites establecidos por el propio mercado y, por otra, lo que Habermas postuló como "el fin de la esfera pública burguesa y la constitución de un sistema de manipulación de las conciencias" (citado en Sierra, Mastrini y Bolaño, 2005: 42).

En este sentido, no es extraño observar que el 64,9 por ciento de los 231 líderes latinoamericanos consultados por el PNUD (2004) perciben los medios de comunicación social como agentes que ostentan un poder y posición de relevancia en el campo de las relaciones sociales dentro de los diversos países. Además, se hace hincapié en el aumento de herramientas de control social, como es el caso de los medios de comunicación, que limitan el poder de las instituciones gubernamentales. En este caso, en el informe se especifica que los líderes creen que la existencia de medios de comunicación independientes es un factor que ha contribuido al aumento de los controles. 
¿Quiénes ejercen poder en América Latina?

Según menciones de los líderes consultados

\begin{tabular}{|c|c|c|c|}
\hline & & $\begin{array}{l}\text { Cantidad de } \\
\text { menciones }\end{array}$ & $\begin{array}{l}\% \text { de consultados } \\
\text { que mencionan }\end{array}$ \\
\hline Poderes fácticos & $\begin{array}{l}\text { Los grupos económicos / empresarios / El sector financiero } \\
\text { Los medios de comunicación }\end{array}$ & 150 & $(79,8 \%)$ \\
\hline Poderes constitucionales & $\begin{array}{l}\text { Poder Ejecutivo } \\
\text { Poder Legislativo } \\
\text { Poder Judicial }\end{array}$ & $\begin{array}{l}68 \\
24 \\
16\end{array}$ & $\begin{array}{c}(36,2 \%) \\
(12,8 \%) \\
(8,5 \%)\end{array}$ \\
\hline Fuerzas de seguridad & $\begin{array}{l}\text { Las Fuerzas Armadas } \\
\text { La Policía }\end{array}$ & $\begin{array}{c}40 \\
5\end{array}$ & $\begin{array}{c}(21,3 \%) \\
(2,7 \%)\end{array}$ \\
\hline $\begin{array}{l}\text { Instituciones políticas y } \\
\text { líderes políticos }\end{array}$ & $\begin{array}{l}\text { Partidos políticos } \\
\text { Los políticos / operadores políticos / líderes políticos }\end{array}$ & $\begin{array}{l}56 \\
13\end{array}$ & $\begin{array}{c}(29,8 \%) \\
(6,9 \%)\end{array}$ \\
\hline Factores extraterritoriales & $\begin{array}{l}\text { EE.UU. / La embajada norteamericana } \\
\text { Organismos multilaterales de crédito } \\
\text { el factor internacional / el factor externo } \\
\text { Empresas transnacionales / multinacionales }\end{array}$ & $\begin{array}{c}43 \\
31 \\
13 \\
9\end{array}$ & $\begin{array}{c}(22,9 \%) \\
(16,5 \%) \\
(6,9 \%) \\
(4,8 \%)\end{array}$ \\
\hline
\end{tabular}

Nota: $\mathrm{n}=188$. El total no suma $100 \%$ porque se permitieron respuestas múltiples.

Fuente: PRODDAL, Ronda de consultas con líderes de América Latina, 2002.

Informe PNUD, 2004, p. 155

Entonces, si planteamos que la concentración económica de las empresas informativas genera una dependencia de éstas con los centros económicos y políticos dominantes ces posible la existencia de medios de comunicación independientes que contribuyan al control del Estado y de los poderes fácticos como es el caso de los diversos grupos económicos presentes en América Latina?

La afirmación formulada por Mastrini y Becerra es categórica:

El alto grado de concentración de la propiedad de los medios, en manos de las elites económicas y políticas, han clausurado hasta ahora las opciones de un desarrollo más democrático. La concentración de la riqueza en muy pocas manos marca que este, desgraciadamente, no es sólo un problema del sector audiovisual (2001: 206).

\section{Una aproximación a la concentración de los medios de comunicación en Chile}

Los procesos de concentración en el sector de las industrias culturales, específicamente en el sector de los medios de comunicación, denota una tendencia oligopólica de los actores mediáticos con el fin de reorganizar en virtud de sus propios intereses el espacio público. Este fenómeno de concentración, además, se ve acelerado por el fenómeno de la globalización y la circulación de un mayor volumen de capital transnacional en búsqueda de nuevos sectores productivos de inversión.

Así, entonces, en el caso de la concentración mediática en Chile, a partir de los datos obtenidos por el Instituto Prensa y Sociedad (IPyS) como resultado de una investigación desarrollada entre el 2002 y 2005, se puede observar lo siguiente:

\subsection{Chile}

A partir de las medidas tomadas por el gobierno militar durante la década del 80, la lógica de la privatización y la liberalización de la economía produjeron, por una parte, un aumento de las desigualdades ${ }^{1}$ en la distribución de las riquezas (situación que se mantiene en la actualidad a pesar del descenso en los niveles de pobreza entre los años 1990 y 2006) y, por otra, la disminución del poder del Estado en el marco de las reglas esta-

\footnotetext{
"Es innegable que la distribución del ingreso autónomo continúa siendo altamente desigual en el país, con el 20\% de hogares de mayores ingresos aún concentrando más del $50 \%$ del total de ingresos autónomos, en tanto que el 20\% de hogares de menores ingresos reciben sólo el 4,1\% de estos ingresos" (CASEN, 2006: 6). Consultar: http://www.mideplan.cl/casen/
} 
blecidas por el mercado como agente promotor de la libre competencia.

En esta realidad los medios de comunicación presentan una alta concentración pero con ausencia de la conformación de grandes conglomerados multimedia, al igual que los existentes en otros países de América Latina. No obstante, la intervención de un Estado para promover una mayor pluralidad de contenidos y productos culturales diversos se debe establecer sobre la base de dos aspectos fundamentales para una configuración coherente de un régimen democrático:

1. Establecimiento de un sistema legal que abogue por limitar la concentración de la propiedad de los medios de comunicación en un grupo disminuido de inversionistas.

2. Planificación y establecimiento de políticas públicas asociadas al ámbito de la información, la comunicación y la cultura. Esto permitiría focalizar una determinada cantidad de capital para financiar la creación de empresas ligadas al sector de la industria cultural (local y nacional) y que, por su carácter ciudadano-integrador, sean una alternativa independiente de los principales grupos mediáticos.

Hay más que suficiente evidencia empírica (Mastrini y Becerra, 2006, Sunkel y Geoffroy, $2001)$ para establecer que a partir de la medición del primer operador ${ }^{2}$ dentro del sector infocomunicacional en Chile, los niveles de concentración establecidos en el área de los medios de comunicación social se encuentran entre el 25 y 45 por ciento. (Ver gráfica superior derecha)

Asimismo, si observamos los niveles de participación de los cuatro primeros operadores en el mercado infocomunicacional en Chile, nos damos cuenta de que la estimación estadística a partir de los niveles de facturación y cuota de mercado

2 Actor empresarial que posee una participación (alta, media o baja) dentro del mercado. Cuando se hace referencia al primer operador dentro del análisis de la concentración de los medios de comunicación social en Chile, estamos identificando al actor empresarial que tiene una alta participación (dominio) en el sector productivo infocomunicacional.
Chile: indice de concentración, dominio del primer operador

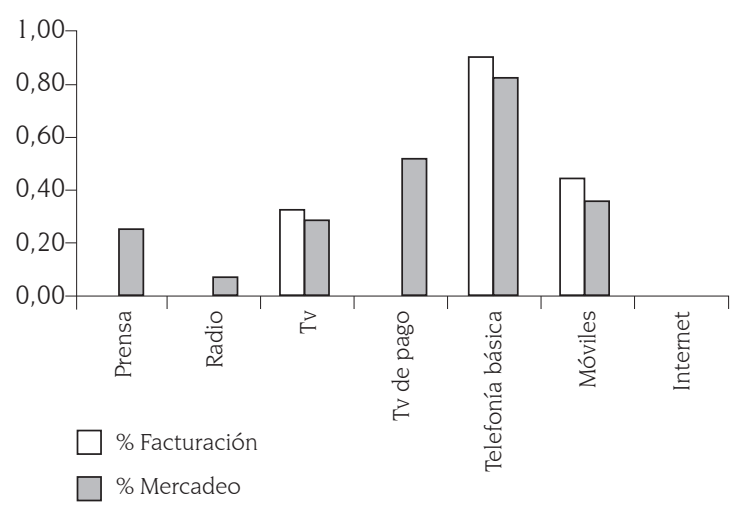

Fuente: Mastrini y Becerra, 2006:164.

fluctúa entre el 70 y 90 por ciento en el caso de la prensa escrita y la televisión (Acceso Público).

Chile: indice de concentración, dominio de los 4 primeros operadores

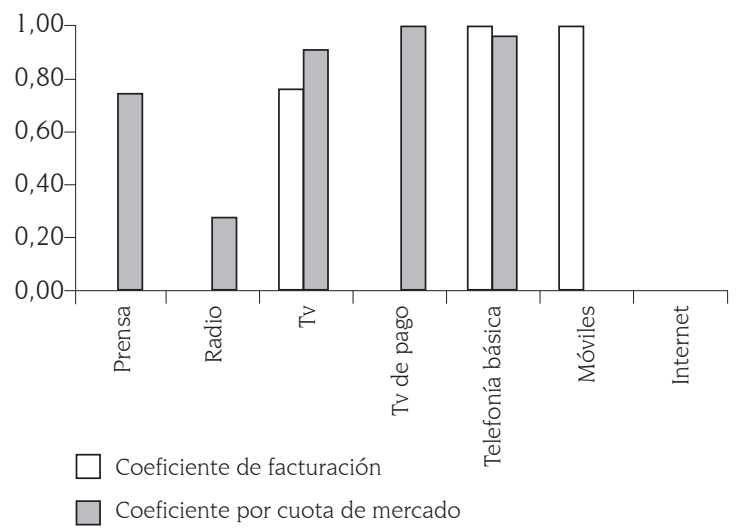

Fuente: Mastrini y Becerra, 2006:165.

Por otra parte, los niveles de concentración observables en el sector de la televisión por pago son cercanos al 100 por ciento. El único contrapeso real pero deficiente que tiene la empresa VTR GlobalCom ${ }^{3}$ en el marco de una posible competencia dentro del mercado de los servicios de TV Cable se encuentra focalizado a nivel regional. Sin embargo, los niveles de participación dentro del

3 En el año 2005 la empresa VTR GlobalCom S.A. (VTR) anunció su fusión con Metrópolis Intercom S.A. (Metrópolis). Dicha fusión le permite a VTR fortalecer su posición dentro del mercado, donde a partir de las cifras entregadas el porcentaje de participación en el mercado ascenderá a 87 por ciento del total. 
mercado regional por parte de algunas empresas locales de TV Cable son minoritarios y no representan una competencia desequilibrante.

\begin{tabular}{|l|l|l|l|c|}
\hline \multicolumn{1}{|c|}{ Grupo } & $\begin{array}{c}\text { Facturación (en } \\
\text { millones US\$̦) }\end{array}$ & $\begin{array}{c}\text { Porcentaje } \\
\text { de } \\
\text { facturación }\end{array}$ & $\begin{array}{c}\text { Cantidad de } \\
\text { abonados* }\end{array}$ & $\begin{array}{c}\text { Porcentaje } \\
\text { de mercado }\end{array}$ \\
\hline VTR & & & 413.037 & $52 \%$ \\
\hline Metrópolis-Intercom & & & 271.037 & $35 \%$ \\
\hline Sky (satélite) & & & 90.721 & $12 \%$ \\
\hline Direct TV (satélite) & & & 9.667 & $1 \%$ \\
\hline $\begin{array}{l}\text { Subtotal 4 operadores } \\
\text { principales }\end{array}$ & & & 787.961 & $100 \%$ \\
\hline Total del mercado & 248.000 .000 & & 787.961 & $100 \%$ \\
\hline $\begin{array}{l}\text { Razón de concentra- } \\
\text { ción de facturación (C) }\end{array}$ & & & & \\
\hline $\begin{array}{l}\text { Razón de concentra- } \\
\text { ción de audiencia }\left(C_{a}\right)\end{array}$ & & & & \\
\hline
\end{tabular}

* Fuente: Mastrini y Becerra, 2006:160.

En el caso específico del sector productivo asociado a la prensa escrita en Chile, los actores empresariales que ejercen un dominio importante en el mercado de la producción y distribución de información periodística son: grupo Edwards (El Mercurio y Las Últimas Noticias) y grupo Copesa (La Tercera y La Cuarta).

\begin{tabular}{|l|l|l|l|l|}
\hline \multicolumn{1}{|c|}{ Grupo } & $\begin{array}{c}\text { Facturación (en } \\
\text { millones US\$) }\end{array}$ & $\begin{array}{c}\text { Porcentaje } \\
\text { de } \\
\text { facturación }\end{array}$ & $\begin{array}{c}\text { Ejemplares } \\
\text { vendidos* }\end{array}$ & $\begin{array}{c}\text { Porcentaje } \\
\text { de } \\
\text { circulación }\end{array}$ \\
\hline El Mercurio & & & 90.375 .600 & $25,97 \%$ \\
\hline La Tercera & & & 69.147 .600 & $19,87 \%$ \\
\hline $\begin{array}{l}\text { Las Últimas } \\
\text { Noticias }\end{array}$ & & 57.141 .600 & $16,42 \%$ \\
\hline La Cuarta & & 43.604 .400 & $12,53 \%$ \\
\hline $\begin{array}{l}\text { Subtotal 4 diarios } \\
\text { principales }\end{array}$ & & 260.269 .200 & $75,08 \%$ \\
\hline Total del mercado & $169.090 .000 *$ & & 348.000 .000 & $100 \%$ \\
\hline $\begin{array}{l}\text { Razón de concen- } \\
\text { tración de factura- } \\
\text { ción (C) }\end{array}$ & & & & \\
\hline $\begin{array}{l}\text { Razón de concen- } \\
\text { tración de audien- } \\
\text { cia (C) }\end{array}$ & & & & \\
\hline
\end{tabular}

* Fuente: Mastrini y Becerra, 2006:157.

Ambos holdings editores tienen una presencia dominante en el mercado, además, son los responsables de las publicaciones periodísticas que poseen una cobertura nacional y, a su vez, concentran un alto porcentaje de los ejemplares vendidos en el ámbito nacional y, en consecuencia, obtienen altos ingresos en virtud del nivel de facturación en publicidad que existe en el mercado chileno.

\section{Chile: facturación por publicidad}

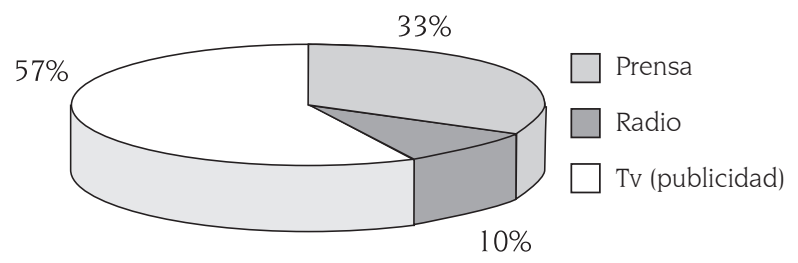

Fuente: Mastrini y Becerra, 2006:155.

Ante esta situación, la lógica de la concentración de los medios de comunicación social en Chile apunta a mejorar los procesos de control de información, fortalecer la posición en el mercado, incrementar el poder económico y político, estandarizar los contenidos y aumentar los ingresos mediante el consumo masivo de los productos culturales que circulan en el mercado interno.

\section{Consideraciones finales}

Apocalípticamente anunciado por Horkheimer y Adorno en Dialéctica del Iluminismo el mercado mediático tendió, desde la década del setenta (situación más patente en Chile), hacia la masificación de sus productos y la estandarización de contenidos, con un desplome de todo lo que huele a crítica social.

En este sentido, si tomamos globalmente el período 1970-2005 y cruzamos de manera general las dimensiones de las estructuras económica, política y mediática, observaremos lo siguiente en relación con el mercado de las comunicaciones en Chile:

1. La existencia de diversos proyectos económicos y sociopolíticos, pues se observa un claro proceso de continuidad, especialmente en el plano político, puesto que, en general: "La atención de las autoridades se ha concentrado en el mantenimiento de los equilibrios macroeconómicos y en profundizar la expansión exportadora de la economía chilena" 
(Alarcón y Stumpo, 2002: 139). Así entonces, el fenómeno de la concentración económica es particularmente preocupante en Chile:

La propiedad del país Chile está compartida entre pocos nacionales y algunos extranjeros [donde] el 10\% más pobre accede sólo al 1,3\% del ingreso, mientras el 10\% más rico logra el $45,6 \%$ [y] el 20\% chileno más rico posee el $61 \%$ del ingreso, contra el 3,3\% del más pobre (Carmona, 2002: 65 y 239).

Esta concentración económica se mantiene, tendiendo a agudizarse, y se manifiesta muy claramente tanto en el caso de la propiedad de los medios de comunicación masiva, como de sus contenidos. A ello se agregan las escasas experiencias de investigación existentes sobre estos procesos de concentración de los medios, pues la preocupación de los investigadores tiende a seguir la lógica del mercado, especialmente del mercado de la publicidad: una excesiva preocupación por el análisis de la incorporación de productos de comunicación mediática y cómo estos influyen en los consumidores; es decir, estudios de audiencia que responden más a intereses de los propietarios de los medios que a una preocupación sociocomunicativa y sociopolítica crítica. De hecho, lo que comenzó como experiencias de comunicación educativa (educomunicación) o pedagogía de la comunicación durante la Reforma Educacional en Chile, orientada a crear consumidores críticos de medios, se transformó rápidamente en una experiencia funcional al mercado, en la cual participan directamente, como auspiciantes, empresas periodísticas del Grupo Edwards, como El Mercurio, y universidades privadas. Los alcances críticos de una comunicación educativa o una pedagogía de la comunicación hoy se conocen como "Prensa en la Escuela" o "Periodismo Escolar", con énfasis en una lógica funcional a los medios (formación de lectores) o de captación de potenciales alumnos (en el caso de las universidades privadas).

2. Un fuerte componente ideológico, pues efectivamente, como sostienen Sunkel y Geoffroy:

La peculiaridad del caso chileno es que estos procesos económicos [de concen- tración] van acompañados de un marcado 'monopolio ideológico' ly] la presencia de grupos extranjeros que se mueven en la lógica de la 'industria de la entretención', van copando los espacios comunicativos y relegando a los márgenes la diversidad cultural y política (2001: 114-115).

3. Una fuerte intervención del Estado-nación, en el sentido no de des-regular, sino de re-regular el mercado de los medios. Aunque prevalecen discusiones sobre el rol asumido por el Estado-nación, especialmente durante la dictadura militar, parece evidente que, existiendo matices, el rol del Estado-nación tiende a la intervención, siguiendo un modelo neoliberal, particularmente a partir de 198384 (Alarcón y Stumpo, 2002).

De hecho, la dictadura militar generó profundas transformaciones estructurales en el medio televisivo, las cuales se observan tanto en el financiamiento como en la participación.

De tal manera que la intervención del Estado-nación chileno durante la Dictadura Militar acabó con la televisión "no comercial", cultural, educativa y creativa que había instituido el gobierno de Salvador Allende, a través de la Ley No 17.377 (Piñuel, 1992: 14).

El nuevo escenario establece un sistema televisivo administrativamente vertical y centralista con una mínima expresión de la sociedad chilena en el acceso, con una pérdida del diálogo, la pluralidad y la crítica en sus contenidos y transforma a la televisión, en general, en un dispositivo publicitario y de persuasión política (Piñuel, 1992: 15-16).

Corresponden al proceso antes descrito de intervención económico-productiva, la eliminación de la prensa política al comenzar la dictadura, la cual "deja a los medios que conformaban el núcleo oligopólico sin competencia posible y, con ello, se va conformando un sistema en el que dos conglomerados lideran el mercado del sector" (Sunkel y Geoffroy, 2001: 31).

Y en términos de estructura económica, la televisión se comercializa y la prensa disminuye drásticamente (de 11 a 5 periódicos), para dejar finalmente dos grandes consorcios. 
A su vez, la dictadura militar interviene en los contenidos, provocando una disminución sustantiva de los programas culturales y educativos, una visión parcial fragmentada y distorsionada de la información y una reducción importante de los contenidos de carácter político (Piñuel, 1992: 16).

4. La inexistencia de políticas comunicacionales, especialmente en el incentivo de medios locales. En efecto, las actuaciones del Estado-nación durante este amplio período (1970-2004) corresponden a medidas políticas para reforzar la estructura de los medios y estrechar el vínculo entre la estructura de los medios y las estructuras de poder político y económico.

5. El bajo nivel de descentralización económica, política y territorial, puesto que se observa un proceso de centralización, al menos, en dos ámbitos:

a) Territorial, pues la mayor parte de los medios pertenecen a reducidos grupos de carácter oligopólico que, desde la capital del país (Santiago) controlan el mercado de la prensa (dos grandes consorcios y un diario del gobierno de turno), la radio (dos consorcios extranjeros, siete radios institucionales y tres empresarios nacionales), y la televisión (seis canales de difusión nacional, dos macro-regionales, nueve canales locales y dos canales de cable). De todos ellos, sólo una cadena de radiodifusión ha surgido en una región, aunque su lógica de administración y gestión es similar a las cadenas nacionales; y excepto la lógica de algunos diarios regionales, se trata de una estructura político-administrativa, económica y programática centrada cada vez con más fuerza en la capital, en la cual se toman las decisiones significativas. Se insiste en lo de algunos diarios regionales, pues durante los últimos años la cadena El Mercurio S.A., compró varios periódicos de carácter regional y local. Pensemos sólo que el Grupo Edwards, uno de los dos consorcios periodísticos en Chile, controla cerca del cincuenta por ciento de los diarios pertenecientes a la Comisión de Diarios Regionales.

b) Económica y política, aunque existen medios locales y zonales se observa una centralización económica y política, pues estos se integran plenamente a los grandes grupos señalados (como cadenas de radiodifusión, diarios regionales o programación envasada), con una excesiva concentración de las decisiones. Estas lógicas de centralización localizada, responden al fuerte proceso de satelización ${ }^{4}$.

Se expresa [la centralización] en el carácter dominante que han adquirido las empresas que se desarrollan desde la Región Metropolitana para expandirse hacia otras regiones del país [y] cada vez es más fuerte la presencia de audiencia en regiones de radios que pertenecen a grandes consorcios" (Sunkel y Geoffroy, 2001: 116)

En consecuencia, al observar la dinámica de la concentración de la propiedad mediática en Chile, es de suma importancia identificar, por una parte, la relevancia y valor local que aún mantiene la producción televisiva de carácter regional, aunque durante el último año existe una tendencia creciente a crear espacios regionales, especialmente noticiosos, dentro de las programaciones de la televisión de carácter nacional, lo cual puede variar el actual escenario. Es decir, habrá mayor producción regional en las cadenas nacionales, lo que podría generar un proceso de cierre de las cadenas de carácter regional a raíz de la falta de recursos económicos, técnicos y humanos necesarios para poder competir con las producciones nacionales. Y, por otra, la importancia de las radios

\footnotetext{
Noción que se utiliza genéricamente, en el caso de las radios, para explicar el proceso gradual de pérdida de la producción local de los medios mediante la incorporación a los grandes conglomerados. Se inició con la compra y fusión de los medios locales, para luego reducir paulatinamente la programación local, hasta eliminarla por completo. Esta lógica, iniciada a fines de la década de los '90, se fundamenta principalmente en la relación con el mercado publicitario: mayor concentración = mayor cobertura; mayor cobertura = mayor auspicio; mayor auspicio $=$ mayores ingresos.
} 
a nivel regional, situación que debe ser aprovechada para aumentar la participación social. Sin embargo, en este caso también se observa una tendencia creciente a la adquisición (compra) de las radios locales por parte de las cadenas trans-nacionales.

\section{Referencias bibliográficas}

Alarcón, Cecilia y Stumpo, Giovanni. (2002). Las pequeñas y medianas empresas industriales en Chile. En: Peres, W. y Stumpo, G. (Coords.). Pequeñas y medianas empresas industriales en América Latina y el Caribe. México: Siglo XXI / CEPAL.

Carmona, Ernesto. (2002). Los dueños de Chile. Santiago de Chile: Ediciones La Huella.

Lechner, Norbert. (2007). Obras escogidas. Vol. 2. Santiago de Chile: LOM.

Mastrini, Guillermo y Becerra, Martín. (2001). 50 años de concentración de medios en América Latina: del patriarcado artesanal a la valorización en escala. En Quirós, Fernando y Sierra, Francisco. (eds.). Globalización, comunicación y democracia. Crítica de la economía política de la comunicación y la cultura. Sevilla: Comunicación Social Ediciones y Publicaciones.

. (2006). Periodistas y magnates. Estructura y concentración de las industrias culturales en América Latina. Prometeo e IPyS ediciones. Buenos Aires.

Mideplan. Encuesta CASEN 2006 (2006). Informe acerca de distribución del ingreso e impacto distributivo del gasto. Disponible en http://www. mideplan.cl/casen/

Piñuel, José Luis. (1992). La cultura política del ciudadano y la comunicación política en TV en la transición política del plebiscito chileno. Madrid: CEDEAL.

PNUD. (2004). La democracia en América Latina. Hacia una democracia de ciudadanas y ciudadanos. Buenos Aires: Alfaguara.

Sartori, Giovanni. (1998). Hommo Videns. La sociedad teledirigida. Madrid: Taurus. . (2002). Elementos de teoría política. Madrid: Editorial Alianza.

Sierra, Francisco, Mastrini, Guillermo y Bolaño, César. (2005). Economía política, comunicación y conocimiento. Una perspectiva crítica latinoamericana. Buenos Aires: La Crujía.

Sunkel, Guillermo y Geoffroy, Esteban. (2001). La concentración económica de los medios de comunicación en Chile. Santiago de Chile: LOM.

Torres López, Juan. (1985). Economía de la comunicación de masas. Madrid: Grupo Cultural Zero.

Zallo, Ramón. (1992). El mercado de la cultura. Estructura económica y política de la comunicación. Navarra: Tercera Prensa. 
